K. Tahara

Nagoya Math. J.

Vol. 33 (1968), 173-194

\title{
ON THE FORMAL THETA FUNCTIONS OF COUNTABLY MANY VARIABLES
}

\author{
KENICHI TAHARA
}

\section{Introduction}

In the previous paper [6] we have shown the examples of hyperelliptic Riemann surfaces of infinite genus such that the Riemann's theta functions associated with them are absolutely convergent.

In the present paper we shall study the formal properties of formal theta functions of countably many variables, analogeous to the case of finite variables [3], [4]: the canonical base and the addition formula etc. .

In $\S 1$, we shall define the formal theta functions of countably many variables with rational characteristics in the same way as [3], [4], and show the formal properties of these functions.

Section 2 is concerned with the special case: the infinite products of the elliptic theta functions with rational characteristics. We shall recall the sufficient conditions under which these theta functions are absolutely convergent and take Jacobi's expression of the elliptic theta functions as infinite products. ${ }^{1)}$ Using this expression we shall give the proofs of our results, which are analogeous to the case of finite variables [3], [4].

The auther expresses his appreciation to Professor Morikawa for his suggestions and encouragements during the preparation of this paper.

\section{Notations and conventions}

$\boldsymbol{Q}^{r}$ : the coordinate vector space of dimension $r$ over the rational number field $\boldsymbol{Q}$,

$\boldsymbol{Z}^{r}$ : the subspace in $\boldsymbol{Q}^{r}$ consisting of all the integral vectors, i.e. vectors with integral coordinates,

$\boldsymbol{Q}^{r} / \boldsymbol{Z}^{r}:$ the residue group of $\boldsymbol{Q}^{r}$ by $\boldsymbol{Z}^{r}$,

Received March 29, 1968.

1) This Jacobi's expression is a generalization of Jacobi's expression of so called Jacobi's theta function $\vartheta_{3}(z, q)$. See p. 464 and 469 in [7]. 
$G_{r}$ : the subgroup $\frac{1}{2} \boldsymbol{Z}^{r} / \boldsymbol{Z}^{r}$ in $\boldsymbol{Q}^{r} / \boldsymbol{Z}^{r}$, i.e. $G_{r}=\left\{\left[\left(\frac{a_{1}}{2}, \frac{a_{2}}{2}, \cdots, \frac{a_{r}}{2}\right)\right]\right.$ $\left.\mid a_{i} \in\{0,1\}(1 \leq i \leq r)\right\}$

$H_{r}^{+}$: the subset in $\boldsymbol{Q}^{r} / \boldsymbol{Z}^{r}$ consisting of all the elements $\left[\left(\frac{a_{1}}{3}, \frac{a_{2}}{3}, \cdots, \frac{a_{r}}{3}\right)\right]$ such that $a_{i} \in\{0,1\}(1 \leq i \leq r)$,

$\Omega$ : the coordinate vector space consisting of all vectors with countably many components in the rational number field $\boldsymbol{Q}$, of which almost all components are zero,

$\Gamma$ : the subspace in $\Omega$ consisting of all the integral vectors,

$\boldsymbol{A}=\Omega / \Gamma:$ the residue group of $\Omega$ by $\Gamma$,

$G$ : the subgroup in $A$ consisting of all the elements $\left[\left(\frac{a_{1}}{2}, \frac{a_{2}}{2}, \ldots \ldots\right)\right]$ such that $a_{i} \in\{0,1\}(i=1,2, \cdots \cdots)$,

$H^{+}$: the subset in $\boldsymbol{A}$ consisting of all the elements $\left[\left(\frac{a_{1}}{3}, \frac{a_{2}}{3}, \cdots \cdots\right)\right]$ such that $a_{i} \in\{0,1\}(i=1,2, \cdots)$,

$\boldsymbol{a}=\left(a_{1}, a_{2}, \cdots\right)$ : the vector in $\Omega$,

$[\boldsymbol{a}]=\left[a_{1}, a_{2}, \cdots \cdots\right]$ : the class of a vector $\boldsymbol{a}=\left(a_{1}, a_{2}, \cdots \cdots\right)$ in $\boldsymbol{A}$,

$\left[\boldsymbol{a}^{+}\right]=\left[a_{1}^{+}, a_{2}^{+}, \cdots \cdots\right]$ : the element in $H^{+}$.

There exists the canonical embedding of the residue group $\boldsymbol{Q}^{r} / \boldsymbol{Z}^{r}$ into the residue group $\boldsymbol{A}$. Therefore we may be considered that $H_{r}^{+}$is the subset in $\mathrm{H}^{+}$.

\section{§1. Formal theta functions of countably many variables with rational characteristics}

We shall first define the formal theta functions of countably many variables with rational characteristics and show the analogeous results to the case of finite variables [3], [4].

1. 1 Denote by $\{W(i ; a), W(j, k ; b) \mid a, b \in \boldsymbol{Q} ; i, j, k=1,2, \cdots \cdots\}$ a system of indeterminates on which rational numbers operate as following:

$$
W(i ; a)^{c}=W(i ; c a), W(j, k ; b)^{c}=W(j, k ; c b) \quad(c \in \mathbf{Q}) .
$$

Let $\boldsymbol{I}$ be the ideal in the polynomial ring $\boldsymbol{Z}[\{W(i ; a), W(j, k ; b) \mid a, b \in \boldsymbol{Q}$; $i, j, k=1,2, \cdots \cdot\}$ generated by 


$$
\begin{array}{r}
W(i, 0)-1, \quad W(j, k ; 0)-1, \quad W(i ; a) W(i ; b)-W(i ; a+b) \\
W(j, k ; a) W(j, k ; b)-W(j, k ; a+b), \quad W(j, k ; a)-W(k, j ; a) \\
(a, b \in \boldsymbol{Q} ; i, j, k=1,2, \cdots) .
\end{array}
$$

Denote by $U_{i}^{a}$ and $Q_{j, k}^{b}$ the images of $W(i ; a)$ and $W(j, k ; b)$ in the residue ring $B=\boldsymbol{Z}[\{W(i ; a), W(j, k ; b) \mid a, b \in \boldsymbol{Q} ; i, j, k=1,2, \cdots\}] / \boldsymbol{I}$, respectively. Then it follows that

$$
\begin{aligned}
& U_{i}^{0}=1, \quad Q_{j, k}^{0}=1, \quad U_{i}^{a} U_{i}^{b}=U_{i}^{a+b} \\
& Q_{j, k}^{a} Q_{j, k}^{b}=Q_{j, k}^{a+b}, \quad Q_{j, k}^{a}=Q_{k, j}^{a} \\
& \quad(a, b \in \boldsymbol{Q} ; i, j, k=1,2, \cdots \cdots) .
\end{aligned}
$$

Using the brief notations

$$
\begin{aligned}
U(\boldsymbol{a})=\prod_{i=1}^{\infty} U_{i}^{a_{i}}, \quad Q(\boldsymbol{a}, \boldsymbol{b})=\prod_{i, j=1}^{\infty} Q_{i, j}^{a_{i} b_{j}} \\
\left(\boldsymbol{a}=\left(a_{1}, a_{2}, \cdots\right), \quad \boldsymbol{b}=\left(b_{1}, b_{2}, \cdots\right) \in \Omega\right),
\end{aligned}
$$

we have the following multiplicative rules:

$$
\begin{gathered}
U(\boldsymbol{a})^{c}=U(c \boldsymbol{a}), \quad Q(\boldsymbol{a}, \boldsymbol{b})^{c}=Q(c \boldsymbol{a}, \boldsymbol{b})=Q(\boldsymbol{a}, c \boldsymbol{b}) \\
U(\boldsymbol{a}) U(\boldsymbol{b})=U(\boldsymbol{a}+\boldsymbol{b}), \quad Q(\boldsymbol{a}+\boldsymbol{b}, \boldsymbol{c}+\boldsymbol{d})=Q(\boldsymbol{a}, \boldsymbol{c}) Q(\boldsymbol{a}, \boldsymbol{d}) Q(\boldsymbol{b}, \boldsymbol{c}) Q(\boldsymbol{b}, \boldsymbol{d}) \\
(c \in \boldsymbol{Q} ; \boldsymbol{a}, \boldsymbol{b}, \boldsymbol{c}, \boldsymbol{d} \in \Omega) .
\end{gathered}
$$

Let $\operatorname{Hom}\left(\Omega, B^{*}\right)$ denote the group of all the homomorphisms of the additive group $\Omega$ into the multiplicative group $B^{*}$ of units in the residue ring $B$. Then $U, U^{-1}$ and $Q(\boldsymbol{a})(\boldsymbol{a} \in \Omega)$ may be regarded as the elements in $\operatorname{Hom}\left(\Omega, B^{*}\right)$ defined by

$$
\begin{aligned}
& U: \boldsymbol{c} \longrightarrow U(\boldsymbol{c}) \\
& U^{-1}: \boldsymbol{c} \longrightarrow U(-\boldsymbol{c}) \\
& Q(\boldsymbol{a}): \boldsymbol{c} \longrightarrow Q(\boldsymbol{a})(\boldsymbol{c})=Q(\boldsymbol{a}, \boldsymbol{c}) \quad(\boldsymbol{c} \in \Omega) .
\end{aligned}
$$

The products $Q(\boldsymbol{a}) U(\boldsymbol{a} \in \Omega)$ and $Q(\boldsymbol{a}) Q(\boldsymbol{b})(\boldsymbol{a}, \boldsymbol{b} \in \Omega)$ mean the elements in $\operatorname{Hom}\left(\Omega, B^{*}\right)$ defined by

$$
\begin{aligned}
& Q(\boldsymbol{a}) U: \boldsymbol{c} \longrightarrow Q(\boldsymbol{a}, \boldsymbol{c}) U(\boldsymbol{c}) \\
& Q(\boldsymbol{a}) Q(\boldsymbol{b}): \boldsymbol{c} \longrightarrow Q(\boldsymbol{a}, \boldsymbol{c}) Q(\boldsymbol{b}, \boldsymbol{c})=Q(\boldsymbol{a}+\boldsymbol{b}, \boldsymbol{c}) \quad(\boldsymbol{c} \in \Omega) .
\end{aligned}
$$

1. 2 In the same way as [4], the theta functions with rational characteristics are defined as follows 


$$
\vartheta_{[a]}(Q \mid U)=\sum_{m \in \Gamma} Q(\boldsymbol{m}+\boldsymbol{a}, \boldsymbol{m}+\boldsymbol{a}) U(\boldsymbol{m}+\boldsymbol{a})^{2} \quad([\boldsymbol{a}] \in \boldsymbol{A}) .
$$

The function $\vartheta_{[a]}(Q \mid U)$ does depend on the choice of the representative $\boldsymbol{a}$ of [a]. The theta zero values are also defined by

$$
\vartheta_{[\boldsymbol{a}]}(Q)=\sum_{\boldsymbol{m} \in \Gamma} Q(\boldsymbol{m}+\boldsymbol{a}, \boldsymbol{m}+\boldsymbol{a}) \quad([\boldsymbol{a}] \in \boldsymbol{A}) .
$$

The theta zero value $\vartheta_{[a]}(Q)$ is regarded as the specialization of $\vartheta_{[a]}(Q \mid U)$ with respect to the replacement of $U_{i}^{a}(a \in \boldsymbol{Q} ; i=1,2, \cdots)$ by 1 .

From the definitions (1. 1) and (1.2) we have the following formulae:

$$
\begin{array}{ll}
(1.3) & \vartheta_{[a]}(Q \mid Q(\boldsymbol{l}) U)=Q(\boldsymbol{l}, \boldsymbol{l})^{-1} U(\boldsymbol{l})^{-2} \vartheta_{[\boldsymbol{a}]}(Q \mid U) \quad(\boldsymbol{l} \in \Gamma) \\
(1.4) & \vartheta_{[\boldsymbol{a}]}\left(Q \mid U^{-1}\right)=\vartheta_{-[\boldsymbol{a}]}(Q \mid U) \\
(1.5) & \vartheta_{[\boldsymbol{a}]}(Q \mid Q(\boldsymbol{b}) U)=Q(\boldsymbol{b}, \boldsymbol{b})^{-1} U(\boldsymbol{b})^{-2} \vartheta_{[\boldsymbol{a}]+[\boldsymbol{b}]}(Q \mid U) \quad([\boldsymbol{b}] \in \boldsymbol{A}) \\
(1.6) & \vartheta_{[\boldsymbol{a}]}(Q)=\vartheta_{-[\boldsymbol{a}]}(Q) \\
(1.7) & \vartheta_{[\boldsymbol{a}]+[\boldsymbol{b}]}(Q)=Q(\boldsymbol{b}, \boldsymbol{b}) \vartheta_{[\boldsymbol{a}]}(Q \mid Q(\boldsymbol{b})) \quad([\boldsymbol{b}] \in \boldsymbol{A}) .
\end{array}
$$$$
\text { (1. 6) } \quad \vartheta_{[a]}(Q)=\vartheta_{-[a]}(Q)
$$

The products of $\vartheta_{[\boldsymbol{a}]}(Q \mid U), \vartheta_{[\boldsymbol{b}]}(Q)([\boldsymbol{a}],[\boldsymbol{b}] \in \boldsymbol{A})$ can be defined as the series in $U$ and $Q$, hence the polynomial ring $\boldsymbol{Z}\left[\left\{\vartheta_{[\boldsymbol{a}]}(Q \mid U), \vartheta_{[\boldsymbol{b}]}(Q) \mid[\boldsymbol{a}],[\boldsymbol{b}] \in \boldsymbol{A}\right\}\right]$ is well-defined.

1. 3 For each subset $S$ in $\boldsymbol{A}=\Omega / \Gamma$, denote by $S^{*}$ the inverse image of $S$ by the canonical homomorphism from $\Omega$ onto $A=\Omega / \Gamma$. Let us give the abstract definition of the formal theta functions of variables $U$.

Definition 1. Let $n$ be a natural number and $S$ be a finite subset in $\boldsymbol{A}$. Then a formal series

$$
\varphi(U)=\sum_{\boldsymbol{m} \in S^{*}} \lambda_{\boldsymbol{m}} U(\boldsymbol{m})^{2}
$$

is called a formal theta function of type $(n ; S)$ with coefficients in a ring $R$ if

$$
\lambda_{\boldsymbol{m}} Q(\boldsymbol{m}, \boldsymbol{m})^{-\frac{1}{n}} \in R \quad\left(\boldsymbol{m} \in S^{*}\right)
$$

and $\varphi(U)$ satisfies the difference relation:

$$
\varphi(Q(\boldsymbol{l}) U)=Q(\boldsymbol{l}, \boldsymbol{l})^{-n} U(\boldsymbol{l})^{-2 n} \varphi(U) \quad(\boldsymbol{l} \in \Gamma) .
$$

We mean by $\boldsymbol{M}_{R}(n ; S)$ the $R$-module of formal theta functions of type $(n ; S)$ with coefficients in the ring $R$. 
It is easy to show that $\vartheta_{[\boldsymbol{a}]}(Q \mid U)$ belongs to $\boldsymbol{M}_{R}(1 ;[\boldsymbol{a}])$, where $R$ is any ring containing the identity 1.

By the same computation as [4] we have

Proposition 1. Let $n$ be a natural number and $S$ be a finite subset in $\boldsymbol{A}$. Then for any field $K, \vartheta_{\left[\boldsymbol{b}_{i}\right]}\left(Q^{n} \mid U^{n}\right)\left(\left[\boldsymbol{b}_{i}\right] \in \frac{1}{n} S\right)$ form a base of $\boldsymbol{M}_{K}(n ; S)$, where $\frac{1}{n} S$ is the subset $\{[\boldsymbol{b}] \in \boldsymbol{A} \mid n[\boldsymbol{b}] \in S\}$ in $\boldsymbol{A}$.

1. 4 We denote by $\Lambda$ the graded ring $\boldsymbol{Z}\left[\left\{\vartheta_{[a]}(Q) \mid[\boldsymbol{a}] \in \boldsymbol{A}\right\}\right]$ and define the formal theta functions of variables $U \otimes_{A} V$.

Definition 2. Let $m$ and $n$ be two natural numbers, and $S$ and $T$ be two finite subsets in $\boldsymbol{A}$. Then a formal theta functions of type $(m \times n ; S \times T)$ with coefficients in a ring $R$ is a formal series

$$
\varphi\left(U \otimes_{A} V\right)=\sum_{\substack{\boldsymbol{m} \in S^{*} \\ \boldsymbol{n} \in T^{*}}} \lambda_{\boldsymbol{m}, \boldsymbol{n}} U(\boldsymbol{m})^{2} \otimes_{\Lambda} V(\boldsymbol{n})^{2}
$$

such that

$$
\lambda_{\boldsymbol{m}, \boldsymbol{n}} Q(\boldsymbol{m}, \boldsymbol{m})^{-\frac{1}{m}} Q(\boldsymbol{n}, \boldsymbol{n})^{-\frac{1}{n}} \in R \quad\left(\boldsymbol{m} \in S^{*}, \boldsymbol{n} \in T^{*}\right)
$$

and satisfies the difference relation

$$
\begin{gathered}
\varphi\left(Q(\boldsymbol{k}) U \otimes_{A} Q(\boldsymbol{l}) V\right)=Q(\boldsymbol{k}, \boldsymbol{k})^{-m} Q(\boldsymbol{l}, \boldsymbol{l})^{-n} U(\boldsymbol{k})^{-2 m} \otimes_{A} V(\boldsymbol{l})^{-2 n} \varphi\left(U \otimes_{A} V\right) \\
(\boldsymbol{k}, \boldsymbol{l} \in \Gamma) .
\end{gathered}
$$

Let $\varphi(U)=\sum_{m \in S^{*}} \lambda_{m} U(\boldsymbol{m})^{2}$ be a formal theta functions of type $(n ; S)$ with coefficients in a ring $R$. Put

$$
\varphi\left(U \otimes_{\Lambda} V\right)=\sum_{\substack{\boldsymbol{m} \in S^{*} \\ \boldsymbol{n} \in S^{*}}} \lambda_{\boldsymbol{m}} \lambda_{\boldsymbol{n}} U(\boldsymbol{m})^{2} \otimes_{\Lambda} V(\boldsymbol{n})^{2} .
$$

Then $\varphi\left(U \otimes{ }_{A} V\right)$ is a formal theta function of type $(n \times n ; S \times S)$ with coefficients in $R$.

We shall show the following

Lemma 1. The products $\vartheta_{[\boldsymbol{a}]+[\boldsymbol{b}]}\left(Q \mid U \otimes_{\boldsymbol{A}} V\right) \vartheta_{[\boldsymbol{a}]-[\boldsymbol{b}]}\left(Q \mid U \otimes_{\left.A V^{-1}\right)}([\boldsymbol{a}],[\boldsymbol{b}] \in \boldsymbol{A})\right.$ are formal theta functions of type $(2 \times 2 ; 2[\boldsymbol{a}] \times 2[\boldsymbol{b}])$ with coefficients in any ring $R$ containing the identity 1. 
Proof. From the definition it follows that

$$
\begin{gathered}
\vartheta_{[a]+[b]}\left(Q \mid U \otimes_{A} V\right) \vartheta_{[a]-[b]}\left(Q \mid U \otimes_{A} V^{-1}\right) \\
=\sum_{\boldsymbol{m}, \boldsymbol{n} \in \Gamma} Q(\boldsymbol{m}+\boldsymbol{a}+\boldsymbol{b}, \boldsymbol{m}+\boldsymbol{a}+\boldsymbol{b}) Q(\boldsymbol{n}+\boldsymbol{a}-\boldsymbol{b}, \boldsymbol{n}+\boldsymbol{a}-\boldsymbol{b}) U(\boldsymbol{m}+\boldsymbol{n}+\mathbf{2} \boldsymbol{a})^{2} \otimes_{A} V(\boldsymbol{m}-\boldsymbol{n}+\mathbf{2} \boldsymbol{b})^{2} \\
=\sum_{\boldsymbol{k}, \boldsymbol{l} \in \Gamma} Q\left(\frac{\boldsymbol{k}+\boldsymbol{l}}{\mathbf{2}}+\boldsymbol{a}+\boldsymbol{b}, \frac{\boldsymbol{k}+\boldsymbol{l}}{2}+\boldsymbol{a}+\boldsymbol{b}\right) Q\left(\frac{\boldsymbol{k}-\boldsymbol{l}}{2}+\boldsymbol{a}-\boldsymbol{b}, \frac{\boldsymbol{k}-\boldsymbol{l}}{2}+\boldsymbol{a}-\boldsymbol{b}\right) U(\boldsymbol{k}+\mathbf{2} \boldsymbol{a})^{2} \\
\otimes_{\Lambda} V(\boldsymbol{l}+\mathbf{2} \boldsymbol{b})^{2} .
\end{gathered}
$$

Putting

$$
\vartheta_{[a]+[b]}\left(Q \mid U \otimes_{A} V\right) \vartheta_{[a]-[b]}\left(Q \mid U \otimes_{A} V^{-1}\right)=\sum_{k, l \in \Gamma} \lambda_{k+2 a, l+2 b} U(\boldsymbol{k}+2 \boldsymbol{a})^{2} \otimes_{\Lambda} V(\boldsymbol{l}+2 \boldsymbol{b})^{2}
$$

we have

$$
\lambda_{k+2 a, l+2 b} Q(k+a, k+2 a)^{-\frac{1}{2}} Q(l+2 b, \quad l+2 b)^{-\frac{1}{2}}=1 \in R \quad(k, l \in \Gamma) .
$$

By virtue of the definition 1 and (1.3), it follows that

$$
\begin{gathered}
\left.\vartheta_{[\boldsymbol{a}]+[b]}\left(Q \mid Q(\boldsymbol{k}) U \otimes_{\Lambda} Q(\boldsymbol{l}) V\right) \vartheta_{[\boldsymbol{a}]-[\boldsymbol{b}]}\left(Q \mid Q(\boldsymbol{k}) U \otimes_{\Lambda}(Q(\boldsymbol{l}) V)\right)^{-1}\right) \\
=Q(\boldsymbol{k}, \boldsymbol{k})^{-2} Q(\boldsymbol{l}, \boldsymbol{l})^{-2} U(\boldsymbol{k})^{-4} \otimes_{\Lambda} V(\boldsymbol{l})^{-4} \vartheta_{[\boldsymbol{a}]+[\boldsymbol{b}]}\left(Q \mid U \otimes_{\Lambda} V\right) \vartheta_{[\boldsymbol{a}]-[\boldsymbol{b}]}\left(Q \mid U \otimes_{\Lambda} V^{-1}\right) \\
(\boldsymbol{k}, \boldsymbol{l} \in \Gamma) .
\end{gathered}
$$

Thus we have proved Lemma 1, Q.E.D.

1. 5 We shall show analogeous formal results to the case of finite variables [4]: the practical methods we shall use to prove them are to be expounded in the next section.

Lemma 2. Let $r$ be a natural number. Then

$$
\operatorname{det}\left(\vartheta_{\left[\mathbf{c}^{+}\right]+\left[d^{+}\right]}(Q) \vartheta_{-\left[c^{+}\right]+\left[d^{+}\right]}(Q)\right)_{H_{r}^{+} \times H_{r}^{+}} \not \equiv 0 \bmod . p
$$

for any prime number $p$, and the matrix

$$
\left(\vartheta_{\left[c^{+}\right]+\left[d^{+}\right]}(Q) \vartheta_{-\left[c^{+}\right]+\left[d^{+}\right]}(Q)\right)_{H^{+} \times H^{+}}
$$

is invertible.

Proof. Similarly as Lemma 5 in the next section, we have

$$
\operatorname{det}\left(\prod_{i=1}^{\infty} \vartheta_{\left[c_{i}{ }^{+}\right]+\left[d_{i}{ }^{+}\right]}\left(Q_{i}\right) \prod_{i=1}^{\infty} \vartheta_{-\left[c_{i}{ }^{+}\right]+\left[d_{i}{ }^{+}\right]}\left(Q_{i}\right)\right)_{H_{r}^{+} \times H_{r}^{+}} \not \equiv 0 \bmod . p
$$


for any prime number $p$. Since $\prod_{i=1}^{\infty} \vartheta_{\left[c_{i}^{+}\right]+\left[d_{i}^{+}\right]}(Q)$ is the specialization of $\vartheta_{\left[c^{+}\right]+\left[d^{+}\right]}(Q)$ induced by $Q_{i, j}=1(i \neq j)$ and $Q_{i, i}=Q_{i}(i=1,2, \cdots)$, we have

$$
\operatorname{det}\left(\vartheta_{\left[c^{+}\right]+\left[d^{+}\right]}(Q) \vartheta_{-\left[c^{+}\right]+\left[d^{+}\right]}(Q)\right)_{H_{r}^{+} \times H_{r}^{+}} \not \equiv 0 \text { mod. } p
$$

for any prime number $p$. By virtue of Lemma 7 in the next section and the above same method it follows that the matrix

$$
\left(\vartheta_{\left[c^{+}\right]+\left[d^{+}\right]}(Q) \vartheta_{-\left[c^{+}\right]+\left[d^{+}\right]}(Q)\right)_{H^{+} \times H^{+}}
$$

is invertible, Q.E.D.

Lemma 3. The matrix

$$
\left(\vartheta_{\left[c^{+}\right]-[l]}^{(2)}(Q)\right)_{H^{+} \times G}
$$

is invertible, where $\vartheta_{\left[\boldsymbol{c}^{+}\right]-[l]}^{(2)}(Q)=\sum_{\boldsymbol{m} \in \Gamma} Q\left(\boldsymbol{m}+\boldsymbol{c}^{+}-\boldsymbol{l}, \boldsymbol{m}+\boldsymbol{c}^{+}-\boldsymbol{l}\right)^{2}\left(\left[\boldsymbol{c}^{+}\right] \in H^{+},[\boldsymbol{l}] \in G\right)$.

Proof. The similar computation as Lemma 6 in the next section shows that

$$
\operatorname{det}\left(\prod_{i=1}^{\infty} \vartheta_{\left[c_{i}{ }^{+}\right]-\left[l_{i}\right]}^{(2)}\left(Q_{i}\right)\right)_{H^{+} \times G}=\prod_{i=1}^{\infty} D_{i}
$$

where $D_{i}$ means the determinant of the matrix

$$
\left(\begin{array}{ll}
\vartheta_{[0]}^{(2)}\left(Q_{i}\right), & \vartheta_{\left[\frac{1}{2}\right\rfloor}^{(2)}\left(Q_{i}\right) \\
\vartheta_{\left[\frac{1}{3}\right]}^{(2)}\left(Q_{i}\right), & \vartheta_{\left[\frac{1}{6} j\right.}^{(2)}\left(Q_{i}\right)
\end{array}\right) .
$$

Then it follows that

$$
\operatorname{det}\left(\prod_{i=1}^{\infty} \vartheta_{\left[c_{i}^{+}\right]-\left[t_{i}\right]}^{(2)}\left(Q_{i}\right)\right)_{H^{+} \times G} \not \equiv 0 \bmod . p
$$

for any prime number $p$. Since $\prod_{i=1}^{\infty} \vartheta_{\left[c_{i}^{+}\right]-\left[l_{i}\right]}^{(2)}\left(Q_{i}\right)$ is the specialization of $\vartheta_{\left[c^{+}\right]-[l]}^{(2)}(Q)$ induced by $Q_{i, j}=1(i \neq j)$ and $Q_{i, i}=Q_{i}(i=1,2, \cdots)$ we have

$$
\operatorname{det}\left(\vartheta_{\left[c^{+}\right]-[l]}^{(2)}(Q)\right)_{H^{+} \times G} \not \equiv 0 \bmod . p
$$

for any prime number $p$, which proves Lemma 3, Q.E.D. 
Using the above lemmas, by the same methods as Proposition 5 and 7 in the next section we have

Proposition 2. Let $[\boldsymbol{a}]$ and $[\boldsymbol{b}]$ be in $\boldsymbol{A}$ and $K$ a field such that $\vartheta_{\left[\boldsymbol{c}^{+}\right]+[\boldsymbol{a}]}(\boldsymbol{Q} \mid U) \vartheta_{-\left[\boldsymbol{c}^{+}\right]+[\boldsymbol{a}]}(Q \mid U) \quad\left(\left[\boldsymbol{c}^{+}\right] \in H^{+}\right)$be in $\boldsymbol{M}_{K}(2 ; 2[\boldsymbol{a}])$. Then $\vartheta_{\left[\boldsymbol{c}^{+}\right]+[\boldsymbol{a}]}(Q \mid U)$ $\vartheta_{-\left[\boldsymbol{c}^{+}\right]+[\boldsymbol{a}]}(Q \mid U)\left(\left[\boldsymbol{c}^{+}\right] \in H^{+}\right)$form a base of $\boldsymbol{M}_{K}(2 ; 2[\boldsymbol{a}])$.

Proposition 3. Let [a] and [b] be in $\boldsymbol{A}$ and $K$ a field such that $\vartheta_{\left[c^{+}\right]+[a]}(Q \mid U) \vartheta_{-\left[c^{+}\right]+[a]}(Q \mid U)\left(\left[c^{+}\right] \in H^{+}\right)$and $\vartheta_{\left[d^{+}\right]+[b]}(Q \mid V) \vartheta_{-\left[d^{+}\right]+[b]}(Q \mid V)\left(\left[d^{+}\right] \in H^{+}\right)$ belong to $\boldsymbol{M}_{K}(2 ; 2[\boldsymbol{a}])$ and $\boldsymbol{M}_{K}(2 ; 2[\boldsymbol{b}])$, respectively. Then

$$
\vartheta_{\left[c^{+}\right]+[a]}(Q \mid U) \vartheta_{-\left[c^{+}\right]+[a]}(Q \mid U) \vartheta_{\left[d^{+}\right]+[b]}(Q \mid V) \vartheta_{-\left[d^{+}\right]+[b]}(Q \mid V)\left(\left[c^{+}\right],\left[d^{+}\right] \in H^{+}\right)
$$

form a base of $\boldsymbol{M}_{K}(2 \times 2 ; 2[\boldsymbol{a}] \times 2[\boldsymbol{b}])$.

\section{Theorem (The addition formula)}

$$
\begin{aligned}
& \vartheta_{[\boldsymbol{a}]+[b]}\left(Q \mid U \otimes_{\Lambda} V\right) \vartheta_{[\boldsymbol{a}]-[b]}\left(Q \mid U \otimes_{\Lambda} V^{-1}\right) \\
&= \sum_{\left[c^{+}\right],\left[\boldsymbol{d}^{+}\right] \in H^{+}} \alpha_{\left(\boldsymbol{c}^{+}, \boldsymbol{d}^{+}\right)} \vartheta_{\left[\boldsymbol{c}^{+}\right]+[\boldsymbol{a}]}(Q \mid U) \vartheta_{-\left[c^{+}\right]+[\boldsymbol{a}]}(Q \mid U) \vartheta_{\left[\boldsymbol{d}^{+}\right]+[b]}(Q \mid V) \vartheta_{-\left[\boldsymbol{d}^{+}\right]+[b]}(Q \mid V) \\
&([\boldsymbol{a}],[\boldsymbol{b}] \in \boldsymbol{A})
\end{aligned}
$$

where $\left(\alpha_{\left(\mathbf{c}^{+}, \boldsymbol{d}^{+}\right)}\right)$is the inverse matrix of the matrix ${ }^{2)}$

$$
\left(\vartheta_{\left[c^{+}\right]+\left[d^{+}\right]}(Q) \vartheta_{-\left[c^{+}\right]+\left[d^{+}\right]}(Q)\right)_{H^{+} \times H^{+}} .
$$

We shall obtain certain relations as corollaries of the addition formula. Putting $V_{i}=1(i=1,2, \cdots \cdots)$, we have

Corollary 1.

$$
\begin{aligned}
& \vartheta_{[a]+[b]}(Q \mid U) \vartheta_{[a]-[b]}(Q \mid U) \\
&= \sum_{\left[c^{+}\right],\left[d^{+}\right] \in H^{+}} \alpha_{\left(c^{+}, d^{+}\right)} \vartheta_{\left[d^{+}\right]+[b]}(Q) \vartheta_{-\left[d^{+}\right]+[b]}(Q) \vartheta_{\left[c^{+}\right]+[a]}(Q \mid U) \vartheta_{-\left[c^{+}\right]+[a]}(Q \mid U) \\
&([\boldsymbol{a}],[\boldsymbol{b}] \in \boldsymbol{A}),
\end{aligned}
$$

and

$$
\begin{aligned}
& \vartheta_{[\boldsymbol{a}]}^{2}(Q \mid U) \\
= & \sum_{\left[\boldsymbol{c}^{+}\right],\left[\boldsymbol{d}^{+}\right] \in H^{+}} \alpha_{\left(\boldsymbol{c}^{+}, \boldsymbol{d}^{+}\right)} \vartheta_{\left[\boldsymbol{d}^{+}\right]}^{2}(Q) \vartheta_{\left[\boldsymbol{c}^{+}\right]+[\boldsymbol{a}]}(Q \mid U) \vartheta_{-\left[\boldsymbol{c}^{+}\right]+[\boldsymbol{a}]}(Q \mid U)([\boldsymbol{a}] \in \boldsymbol{A}) .
\end{aligned}
$$

Putting $V_{i}=U_{i}^{-1}(i=1,2, \cdots \cdots)$, we have

2) The existence of the inverse matrix of this matrix is guaranteed by Lemma 2 . 
Corollary 2.

$$
\begin{aligned}
& \vartheta_{[\boldsymbol{a}]+[\boldsymbol{b}]}\left(Q \mid U \otimes_{\Lambda} U^{-1}\right) \vartheta_{[\boldsymbol{a}]-[\boldsymbol{b}]}\left(Q \mid U \otimes_{\Lambda} U\right) \\
&= \sum_{\left[\boldsymbol{c}^{+}\right],\left[\boldsymbol{d}^{+}\right] \in H^{+}} \alpha_{\left(\boldsymbol{c}^{+}, \boldsymbol{d}^{+}\right)} \vartheta_{\left[\boldsymbol{c}^{+}\right]+[\boldsymbol{a}]}(Q \mid U) \vartheta_{-\left[\boldsymbol{c}^{+}\right]+[\boldsymbol{a}]}(Q \mid U) \vartheta_{-\left[\boldsymbol{d}^{+}\right]-[\boldsymbol{b}]}(Q \mid U) \vartheta_{-\left[\boldsymbol{d}^{+}\right]+[\boldsymbol{b}]}(Q \mid U) \\
&([\boldsymbol{a}],[\boldsymbol{b}] \in \boldsymbol{A}) .
\end{aligned}
$$

\section{$\$ 2$ Infinite products of the elliptic theta functions}

This section is concerned with the special case: the infinite products of the elliptic theta functions with rational characteristics. We shall prove the proposition corresponding to dimension theorem for $\boldsymbol{M}_{K}(2 ; 2[\boldsymbol{a}])$ in the case of finite variables and the addition formula in the special case, which show the worth-whileness of the results in the previous section.

2. 1 Let $\tau$ be a complex number of which imaginary part is positive and $z$ be a complex variable, and write $q=e^{\pi_{\sqrt{l-1} \tau}}$ and $u=e^{\pi_{\sqrt{l}-1}}$, so that $|q|<1$. For each element $[a]$ in $\boldsymbol{Q} / \boldsymbol{Z}$ and a natural number $r$, we shall introduce the theta functions $\vartheta_{[a]}^{(r)}(q ; u)$ with rational characteristic $[a]$ :

$$
\vartheta_{[a]}^{(r)}(q ; u)=\sum_{m \in Z} q^{r(m+a)^{2}} u^{2(m+a)} .
$$

Then this function $\vartheta_{[a]}^{(r)}(q ; u)$ does not depend on the choice of the representative $a$ of the class $[a]$ and is absolutely convergent in any bounded domain of values of $z$. In the case $r=1$,

$$
\vartheta_{[a]}^{(1)}(q ; u)=\vartheta_{[a]}(q ; u) \quad([a] \in \boldsymbol{Q} / \boldsymbol{Z})
$$

are the elliptic theta functions with rational characteristics.

Recall the estimations of the these theta functions $\vartheta_{[a]}^{(r)}(q ; u)$. By the same methods as [6], we have

Lemma 4. Let $s$ be the imaginary part of $\tau$, being positive, and $x$ be the imaginary part of $z$. Then

$$
\left|\vartheta_{[a]}^{(r)}(q ; u)\right| \leqslant e^{-\pi a(\text { ras }+2 x)}+\frac{1}{\sqrt{r s}} e^{\frac{\pi x^{2}}{r s}} \quad([a] \in \boldsymbol{Q} / \boldsymbol{Z})
$$

and

$$
\left|\vartheta_{[a]}^{(r)}(q ; u)-1\right| \leq\left|1-e^{-\pi a(\text { ras }+2 x)}\right|+\frac{1}{\sqrt{r s}} e^{\frac{\pi x^{2}}{r s}} \quad([a] \in \boldsymbol{Q} / \boldsymbol{Z}) .
$$




\section{Corollary 1.}

$$
\left|\vartheta_{[0]}^{(r)}(q ; u)\right| \leqslant 1+\frac{1}{\sqrt{r s}} e^{\frac{\pi x^{2}}{r s}}
$$

and

$$
\left|\vartheta_{[0]}^{(r)}(q ; u)-1\right| \leqslant \frac{1}{\sqrt{r s}} e^{\frac{\pi x^{2}}{r s}}
$$

\section{Corollary 2.}

$$
\left|\vartheta_{[0]}^{(r)}(q)\right| \leqslant 1+\frac{1}{\sqrt{r s}}
$$

and

$$
\left|\vartheta_{[0]}^{(r)}(q)-1\right| \leqslant \frac{1}{\sqrt{r s}}
$$

2. 2 Let $\tau_{i}(i=1,2, \cdots \cdots)$ be complex numbers of which imaginary parts $s_{i}$ are positive, and $z_{i}(i=1,2, \cdots)$ be complex variables, and write $q_{i}=e^{\pi \sqrt{-1} \tau_{i}}$ and $u_{i}=e^{\pi \sqrt{-1} z_{i}} \quad(i=1,2, \cdots \cdots)$. For each element $[\boldsymbol{a}]=\left[a_{1}, a_{2}, \ldots.\right]$ in $\boldsymbol{A}$ and a natural number $r$, consider the infinite products of the theta functions $\vartheta_{\left[a_{i}\right]}^{(r)}\left(q ; u_{i}\right)$ with rational characteristic $\left[a_{\imath}\right]$ :

$$
\prod_{i=1}^{\infty} \vartheta_{\left[a_{i}\right]}^{(r)}\left(q_{i} ; u_{i}\right)
$$

In the same way as [6], we have

Proposition 4. Let $s_{i}(i=1,2, \cdots)$ be the imaginary parts of $\tau_{i}$, being positive for all $i$. If the infinite series

$$
\sum_{i=1}^{\infty} \frac{1}{\sqrt{s_{i}}}
$$

is convergent, then the infinite products of the theta functions $\vartheta_{\left[a_{i}\right]}^{(\boldsymbol{r})}\left(q_{i} ; u_{i}\right)$ with rational characteristic $\left[a_{i}\right]$ :

$$
\prod_{i=1}^{\infty} \vartheta_{\left[a_{i}\right]}^{(\boldsymbol{r})}\left(q_{i} ; u_{i}\right) \quad\left([\boldsymbol{a}]=\left[a_{1}, a_{2}, \cdots \cdots\right] \in \boldsymbol{A}\right)
$$

are absolutely convergent in any bounded domain of values of each variable $z_{i}$. 
2. 3 Under the same notations in the above paragraph 2. 2, if the infinite series

$$
\sum_{i=1}^{\infty} \frac{1}{\sqrt{s_{i}}}
$$

is convergent, then it follows that

$$
\begin{aligned}
\prod_{i=1}^{\infty} \vartheta_{\left[a_{i}\right]}\left(q_{i} ; u_{i}\right) & =\sum_{m \in Z^{\infty}} \prod_{i=1}^{\infty} q_{i}{ }^{\left(m_{i}+a_{i}\right)^{2}} \prod_{i=1}^{\infty} u_{i}{ }^{2\left(m_{i}+a_{i}\right)} \\
& =\sum_{m \in \Gamma} \prod_{i=1}^{\infty} q_{i}{ }^{\left(m_{i}+a_{i}\right)^{2}} \prod_{i=1}^{\infty} u_{i}{ }^{2\left(m_{i}+a_{i}\right)}
\end{aligned}
$$

Hence the infinite products $\prod_{i=1}^{\infty} \vartheta_{\left[a_{i}\right]}\left(q_{i} ; u_{i}\right)([\boldsymbol{a}] \in \boldsymbol{A})$ are special cases of the theta functions $\vartheta_{[a]}(Q \mid U)$ defined in $\S 1$, where $Q_{i, j}^{a}=1(i \neq j), Q_{i, i}=q_{i}$ and $U_{i}=u_{i}(a \in \boldsymbol{Q} ; i, j=1,2, \cdots)$.

From now on in this section, we assume that the infinite series

$$
\sum_{i=1}^{\infty} \frac{1}{\sqrt{\frac{s_{i}}{s_{i}}}}
$$

is convergent.

It is easy to show that for each $[\boldsymbol{a}]$ in $\boldsymbol{A}$ the products

$$
\prod_{i=1}^{\infty} \vartheta_{\left[c_{i}^{+}\right]+\left[a_{i}\right]}\left(q_{i} ; u_{i}\right) \prod_{i=1}^{\infty} \vartheta-\left[c_{i}^{+}\right]+\left[a_{i}\right]\left(q_{i} ; u_{i}\right) \quad\left(\left[c^{+}\right] \in H^{+}\right)
$$

are well-defined and formal theta functions of type $(2 ; 2[\boldsymbol{a}])$ with coefficients in a suitable field. Furthermore we shall show the proposition corresponding to the dimension theorem for $\boldsymbol{M}_{K}(2 ; 2[\boldsymbol{a}])$ in the case of finite variables. $\left.{ }^{3}\right)$

Proposition 5. Let $[\boldsymbol{a}]$ be in $\boldsymbol{A}$ and $K$ a field such that

$$
\begin{gathered}
\prod_{i=1}^{\infty} \vartheta_{\left[c_{i}^{+}\right]+\left[a_{i}\right]}\left(q_{i} ; u_{i}\right) \prod_{i=1}^{\infty} \vartheta_{-\left[c_{i}^{+}\right]+\left[a_{i}\right]}\left(q_{i} ; u_{i}\right)\left(\left[\boldsymbol{c}^{+}\right] \in H^{+}\right) \text {be in } \boldsymbol{M}_{K}(2 ; 2[\boldsymbol{\alpha}]) . \quad \text { Then } \\
\prod_{i=1}^{\infty} \vartheta_{\left[c_{i}^{\ddagger}\right]+\left[a_{i}\right]}\left(q_{i} ; u_{i}\right) \prod_{i=1}^{\infty} \vartheta-\left[c_{i}^{+}\right]+\left[a_{i}\right]\left(q_{i} ; u_{i}\right)\left(\left[\boldsymbol{c}^{+}\right] \in H^{+}\right)
\end{gathered}
$$

form a base of $\boldsymbol{M}_{\boldsymbol{K}}(2 ; 2[\boldsymbol{a}])$.

We shall start with a lemma which will be needed for the proof of the proposition.

3) See Proposition 2 in [4]. 
Lemma 5. Let $r$ be a natural number. Then for any prime number $p$ $\operatorname{det}\left(\prod_{i=1}^{\infty} \vartheta_{\left[c_{i}^{+}\right]+\left[d_{i}^{+}\right]}\left(q_{i}\right) \prod_{i=1}^{\infty} \vartheta_{-\left[c_{i}^{+}\right]+\left[d_{i}^{+}\right]}\left(q_{i}\right)\right)_{H_{r}^{+} \times H_{r}^{+}} \neq \equiv 0 \bmod . p$ where $\left(\prod_{i=1}^{\infty} \vartheta_{\left[c_{i}^{+}\right]+\left[d_{i}^{+}\right]}\left(q_{i}\right) \prod^{\infty} \vartheta_{-\left[c_{i}^{+}\right]+\left[d_{i}^{+}\right]}\left(q_{i}\right)\right)_{H_{r}^{+} \times H_{r}^{+}}$is a $2^{r} \times 2^{r}$-matrix of which $\left[c^{+}\right] \times\left[d^{+}\right]-$ -components $\left(\left[\boldsymbol{c}^{+}\right],\left[\boldsymbol{d}^{+}\right] \in H_{r}^{+}\right)$are $\prod_{i=1}^{\infty} \vartheta_{\left[c_{i}^{+}\right]+\left[d_{i}^{+}\right]}\left(q_{i}\right) \prod_{i=1}^{\infty} \vartheta_{-\left[c_{i}^{+}\right]+\left[d_{i}^{+}\right]}\left(q_{i}\right)$.

Proof. From the definition, we have

$$
\begin{aligned}
& \operatorname{det}\left(\prod_{i=1}^{\infty} \vartheta_{\left[c_{i}^{+}\right]+\left[d_{i}^{+}\right]}\left(q_{i}\right) \prod_{i=1}^{\infty}-\left[c_{i}^{+}\right]+\left[d_{i}^{+}\right]\left(q_{i}\right)\right)_{H_{r}^{+} \times H_{r}^{+}} \\
& =\left(\prod_{i=r+1}^{\infty} \vartheta_{[0]}^{2}\left(q_{i}\right)\right)^{2 r} \operatorname{det}\left(\prod_{i=1}^{r} \vartheta_{\left[c_{i}^{+}\right]+\left[d_{i}^{\dagger}\right]}\left(q_{i}\right) \prod_{i=1}^{r} \vartheta_{-\left[c_{i}^{+}\right]+\left[d_{i}^{+}\right]}\left(q_{i}\right)\right) .
\end{aligned}
$$

Since $\prod_{i=r+1}^{\infty} \vartheta_{[0]}^{2}\left(q_{i}\right) \not \equiv 0$ mod.p for any prime number $p$, Lemma 1 in [4] proves the lemma, Q.E.D.

2. 4 We shall give Jacobi's expression for the elliptic theta functions with rational characteristics as infinite products, which is a generalization of Jacobi's expression of so called Jacobi's theta function $\vartheta_{3}(z, q)^{4)}$ and can give not upper estimations only but lower estimations of these theta functions.

Proposition 6 (Jacobi). Let $[\boldsymbol{a}]$ be in $\boldsymbol{Q} / \boldsymbol{Z}$. Then

$$
\vartheta_{[a]}(q ; u)=q^{a^{2}} u^{2 a} \prod_{i=1}^{\infty}\left(1-q^{2 i}\right) \prod_{i=1}^{\infty}\left(1+q^{2 i+2 a-1} u^{2}\right) \prod_{i=1}^{\infty}\left(1+q^{2 i-2 a-1} u^{-2}\right) .
$$

Proof. Consider the product composed of a finite number of factors

$$
F_{m}(u)=\prod_{i=1}^{m}\left(1+q^{2 i+2 a-1} u^{2}\right) \prod_{i=1}^{m}\left(1+q^{2 i-2 a-1} u^{-2}\right) .
$$

This expression developed according to positive and negative powers of $u$ is of the form

$$
F_{m}(u)=A_{0}^{(m)}+\left(A_{1}^{(m)} u^{2}+A_{-1}^{(m)} u^{-2}\right)+\cdots\left(A_{m}^{(m)} u^{2 m}+A_{-m}^{(m)} u^{-2 m}\right) .
$$

Therefore it follows that

$$
A_{m}^{(m)}=q^{m^{2}+2 a m}, \quad A_{-m}^{(m)}=q^{m^{2}-2 a m} .
$$

4) See p. 464 and 469 in [7]. 
The following identity, which may be at once verified,

$$
\left(q^{2 m}+q^{2 a+1} u^{2}\right) F_{m}(q u)=\left(1+q^{2 m+2 a+1} u^{2}\right) F_{m}(u)
$$

gives between two consecutive coefficients $A_{i+1}^{(m)}$ and $A_{i}^{(m)}$ or $A_{-(i+1)}^{(m)}$ and $A_{-i}^{(m)}$ the relations

$$
\begin{aligned}
& A_{i}^{(m)}=\frac{\left(1-q^{2 m+2 a+2}\right)}{q^{2 i+2 a+1}\left(1-q^{2 m-2 i}\right)} A_{i+1}^{(m)} \\
& A_{-i}^{(m)}=\frac{\left(1-q^{2 m+2 i+2}\right)}{q^{2 i-2 a+1}\left(1-q^{2 m-2 i}\right)} A_{-(i+1)}^{(m)} .
\end{aligned}
$$

Thus we have

$$
\begin{aligned}
& A_{i}^{(m)}=q^{i^{2}+2 a i} \frac{\left(1-q^{2 m+2 i+2}\right)\left(1-q^{2 m+2 i+4}\right) \cdots\left(1-q^{4 m}\right)}{\left(1-q^{2}\right)\left(1-q^{4}\right) \cdots\left(1-q^{2 m-2 i}\right)} \\
& A_{-i}^{(m)}=q^{i^{2}-2 a i} \frac{\left(1-q^{2 m+2 i+2}\right)\left(1-q^{2 m+2 i+4}\right) \cdots\left(1-q^{4 m}\right)}{\left(1-q^{2}\right)\left(1-q^{4}\right) \cdots\left(1-q^{2 m-2 i}\right)} .
\end{aligned}
$$

Now put

$$
\begin{aligned}
F(u) & =\lim _{m \rightarrow \infty} F_{m}(u) \\
& =A_{0}+\left(A_{1} u^{2}+A_{-1} u^{-2}\right)+\left(A_{2} u^{4}+A_{-2} u^{-4}\right)+\cdots \cdot
\end{aligned}
$$

Then $F(u)$ is absolutely (and uniformaly) convergent in any bounded domain of values of variables of $z$, and

$$
\begin{aligned}
& A_{i}=\lim _{m \rightarrow \infty} A_{i}^{(m)}=q^{i 2+2 a i}\left(\prod_{j=1}^{\infty}\left(1-q^{2 j}\right)\right)^{-1} \\
& A_{-i}=\lim _{m \rightarrow \infty} A_{-i}^{(m)}=q^{i^{2}-2 a i}\left(\prod_{j=1}^{\infty}\left(1-q^{2 j}\right)^{-1} .\right.
\end{aligned}
$$

Therefore we have

$$
\begin{aligned}
q^{a^{2}} u^{2 a} \prod_{j=1}^{\infty}\left(1-q^{2 j}\right) F(u) & =q^{a^{2}} u^{2 a} \sum_{m \in Z} q^{m^{2}+2 a m} u^{2 m} \\
& =\sum_{m \in Z} q^{(m+a)^{2}} u^{2(m+a)} .
\end{aligned}
$$

This proves Proposition 6, Q.E.D.

In general, consider an infinite product $\prod_{i=1}^{\infty}\left(1+\alpha_{i}\right)$ such that $\alpha_{i}$ are all non-negative real numbers and $\alpha_{i} \longrightarrow 0(i \longrightarrow \infty)$. Then it follows that

$$
\prod_{i=1}^{\infty}\left(1+\alpha_{i}\right) \leq e^{\sum_{i=1}^{\infty} \alpha_{i}}
$$


Furthermore assume that $\alpha_{i}<\frac{1}{2}(i=1,2, \cdots)$. Then it follows that

$$
\prod_{i=1}^{\infty}\left(1-\alpha_{i}\right) \geq e^{-2} \sum_{i=1}^{\infty} \alpha_{i}
$$

Under the same notations in the previous paragraph 2.1, using the above we have the following as corollaries of Proposition 6.

Corollary 1. Let $z$ be in any bounded domain and $c$ denote the maximum of $|u|^{2}$ and $|u|^{-2}$. Then

$$
\left|\vartheta_{[a]}(q ; u)\right| \leq|q|^{a^{2}} c^{a} e^{\beta} \quad \quad([a] \in \boldsymbol{Q} / \boldsymbol{Z}) \text {. }
$$

where

$$
\beta=\frac{|q|^{2}+c\left\{|q|^{2 a+1}+|q|^{-2 a+1}\right\}}{1-|q|^{2}} .
$$

Corollary 2. Let $r$ be a natural number and $|q|^{*}$ denote the maximum of three elements $|q|^{2 r},|q|^{2 r a+r}$ and $|q|^{-2 r a+r}$ where $a$ is an element in $\boldsymbol{Q}$. If $|q|^{*}<\frac{1}{2}$, then

$$
|q|^{r a^{2}} e^{6|q|^{*}} \geq\left|\vartheta_{[a]}^{(r)}(q)\right| \geq|q|^{r a^{2}} e^{-12|q|^{*}} \quad([a] \in \boldsymbol{Q} / \boldsymbol{Z}) .
$$

2. 5 Proof of Proposition 5. Step $1^{\circ}$. Let $J$ be any finite subset of $\mathrm{H}^{+}$. Then we shall show that the subsystem

$$
\left\{\prod_{i=1}^{\infty} \vartheta_{\left[c_{i}^{+}\right]+\left[a_{i}\right]}\left(q_{i} ; u_{i}\right) \prod_{i=1}^{\infty} \vartheta_{-\left[c_{i}^{+}\right]+\left[a_{i}\right]}\left(q_{i} ; u_{i}\right)\right\}_{\left[c^{+}\right] \in J}
$$

of the system $\left\{\prod_{i=1}^{\infty} \vartheta_{\left[c_{i}^{+}\right]+\left[a_{i}\right]}\left(q_{i} ; u_{i}\right) \prod_{i=1}^{\infty} \vartheta_{-\left[c_{i}^{+}\right]+\left[a_{i}\right]}\left(q_{i} ; u_{i}\right)\right\}_{\left[c^{+}\right] \in H^{+}} \quad$ is linearly independent over $K$. There exists a natural number $r$ such that $J \subset H_{r}^{+}$, then by virtue of Lemma 5 we have

$$
\operatorname{det}\left(\prod_{i=1}^{\infty} \vartheta_{\left[c_{i}^{+}\right]+\left[d_{i}^{+}\right]}\left(q_{i}\right) \prod_{i=1}^{\infty} \vartheta_{-\left[c_{i}^{+}\right]+\left[d_{i}^{+}\right]}\left(q_{i}\right)\right)_{H_{r}^{+} \times_{r}^{+}} \not \equiv 0 \bmod . p
$$

for any prime number $p$. From (1. 7) we can show that $\prod_{i=1}^{\infty} \vartheta_{\left[c_{i}^{+}\right]+\left[d_{i}^{+}\right]}\left(q_{i}\right)$ $\prod_{i=1}^{\infty} \vartheta_{-\left[c_{i}^{+}\right]+\left[d_{i}^{+}\right]}\left(q_{i}\right) \quad$ is the specialization of $\prod_{i=1}^{\infty} q_{i}^{\left(d_{i}^{+}-a_{i}\right)^{2}} \prod_{i=1}^{\infty} \vartheta_{\left[c_{i}^{+}\right]+\left[a_{i}\right]}\left(q_{i} ; u_{i}\right)$ $\prod_{i=1}^{\infty} \vartheta-\left[c_{i}^{\dagger}\right]+\left[a_{i}\right]\left(q_{i} ; u_{i}\right)$ induced by the replacement $u_{i}=q_{i}{ }^{\left(d_{i}^{+}-a_{i}\right)}$. Therefore the relation (2. 9) implies that $\left\{\prod_{i=1}^{\infty} \vartheta_{\left[c_{i}^{+}\right]+\left[a_{i}\right]}\left(q_{i} ; u_{i}\right) \stackrel{\infty}{\Pi} \vartheta_{-\left[c_{i}^{+}\right]+\left[a_{i}\right]}\left(q_{i} ; u_{i}\right)\right\}_{\left[\mathbf{c}^{+}\right] \in H_{r}^{+}}$hence 
$\left\{\prod_{i=1}^{\infty} \vartheta_{\left[c_{i}^{+}\right]+\left[a_{i}\right]}\left(q_{i} ; u_{i}\right) \prod_{i=1}^{\infty} \vartheta_{-\left[c_{i}^{+}\right]+\left[a_{i}\right]}\left(q_{i} ; u_{i}\right)\right\}_{\left[c^{+}\right] \in J}$ are linearly independent over $K$. Thus $\prod_{i=1}^{\infty} \vartheta_{\left[c_{i}^{+}\right]+\left[a_{i}\right]}\left(q_{i} ; u_{i}\right) \prod_{i=1}^{\infty} \vartheta_{-\left[c_{i}^{+}\right]+\left[a_{i}\right]}\left(q_{i} ; u_{i}\right)\left(\left[c^{+}\right] \in H^{+}\right)$are linearly independent over $K$.

Step $2^{\circ}$. We shall next show that any element of $\boldsymbol{M}_{K}(2 ; 2[\boldsymbol{a}])$ is a linear combination of $\prod_{i=1}^{\infty} \vartheta_{\left[c_{i}^{+}\right]+\left[a_{i}\right]}\left(q_{i} ; u_{i}\right) \prod_{i=1}^{\infty} \vartheta_{-\left[c_{i}^{+}\right]+\left[a_{i}\right]}\left(q_{i} ; u_{i}\right) \quad\left(\left[c^{+}\right] \in H^{+}\right)$with coefficients in $K$. Since $\prod_{i=1}^{\infty} \vartheta_{\left[\boldsymbol{l}_{i}\right]+\left[a_{i}\right]}\left(q_{i}^{2} ; u_{i}{ }^{2}\right)([\boldsymbol{l}] \in G)$ form a base of $\boldsymbol{M}_{K}(2 ; 2[\boldsymbol{a}])$ by virtue of Proposition 1, it is sufficient to show that the linear transformation matrix of the base $\left\{\prod_{i=1}^{\infty} \vartheta_{\left[l_{i}\right]+\left[a_{i}\right]}\left(q_{i}^{2} ; u_{i}^{2}\right)\right\}_{[l] \in G}$ to the system $\left\{\prod_{i=1}^{\infty} \vartheta_{\left[c_{i}^{+}\right]+\left[a_{i}\right]}\right.$ $\left.\left(q_{i} ; u_{i}\right) \prod_{i=1}^{\infty} \vartheta-\left[c_{i}^{+}\right]+\left[a_{i}\right]\left(q_{i} ; u_{i}\right)\right\}_{\left[c^{+}\right] \in H^{+}}$is invertible. From the definition it follows that

$$
\begin{aligned}
& \prod_{j=1}^{\infty} \vartheta_{\left[c_{i}^{+}\right]+\left[a_{i}\right]}\left(q_{i} ; u_{i}\right) \prod_{i=1}^{\infty} \vartheta_{-\left[c_{i}^{+}\right]+\left[a_{i}\right]}\left(q_{i} ; u_{i}\right) \\
= & \sum_{[l] \in G} \prod_{i=1}^{\infty} \vartheta_{\left[c_{i}^{+}\right]-\left[l_{i}\right]}^{(2)}\left(q_{i}\right) \prod_{i=1}^{\infty} \vartheta_{\left[l_{i}\right]+\left[a_{i}\right]}\left(q_{i}^{2} ; u_{i}^{2}\right)
\end{aligned}
$$

where by (2.7) and (2. 8) it is easy to show that the right hand is absolutely convergent in any bounded domain of values of each variable $z_{i}$. Let $T$ denote the linear transformation matrix. Then we have

$$
T=\left(\prod_{i=1}^{\infty} \vartheta_{\left[c_{i}^{+}\right]-\left[l_{i}\right]}^{(2)}\left(q_{i}\right)\right)_{H^{+} \times G}
$$

and shall show the following:

Lemma 6. The matrix

$$
T=\left(\prod_{i=1} \vartheta_{\left[c_{i}^{+}\right]-\left[l_{i}\right]}^{(2)}\left(q_{i}\right)\right)_{H^{+} \times G} .
$$

is invertible.

Proof. For any natural $i$, put

$$
\begin{aligned}
& \begin{cases}\beta_{(0,0)}^{(i)}=\vartheta_{[0]}^{(2)}\left(q_{i}\right), & \beta_{\left(0, \frac{1}{2}\right)}^{(i)}=\vartheta_{\left[\frac{1}{2}\right]}^{(2)}\left(q_{i}\right) \\
\beta_{\left(\frac{1}{3}, 0\right)}^{(i)}=\vartheta_{\left[\frac{1}{3}\right]}^{(2)}\left(q_{i}\right), & \beta_{\left(\frac{1}{3}, \frac{1}{2}\right)}^{(i)}=\vartheta_{\left[\frac{1}{6}\right]}^{(2)}\left(q_{i}\right)\end{cases} \\
& \begin{cases}\beta_{(0,0)}^{(i) *}=\vartheta_{\left[\frac{1}{6}\right]}^{(2)}\left(q_{i}\right), & \beta_{\left(0, \frac{1}{2}\right)}^{(i)}=-\vartheta_{\left[\frac{1}{2}\right]}^{(i)}\left(q_{i}\right) \\
\beta_{\left(\frac{1}{3}, 0\right)}^{(i) *}=-\vartheta_{\left[\frac{1}{3}\right]}^{(2)}\left(q_{i}\right), & \beta_{\left(\frac{1}{3}, \frac{1}{2}\right)}^{(i) *}=\vartheta_{[0]}^{(2)}\left(q_{i}\right)\end{cases}
\end{aligned}
$$

and 


$$
D_{i}=\operatorname{det}\left(\begin{array}{ll}
\beta_{(0,0)}^{(i)} & \beta_{\left(0, \frac{1}{2}\right)}^{(i)} \\
\beta_{\left(\frac{1}{3}, 0\right)}^{(i)} & \beta_{\left(\frac{1}{3}, \frac{1}{2}\right)}^{(i)}
\end{array}\right) .
$$

Then it follows that

$$
\left\{\begin{array}{l}
\beta_{(0,0)}^{(i) *} \beta_{(0,0)}^{(i)}+\beta_{\left(0, \frac{1}{2}\right)}^{(i)} \beta_{\left(\frac{1}{3}, 0\right)}^{(i)}=D_{i} \\
\beta_{(0,0)}^{(i) *} \beta_{\left(0, \frac{1}{2}\right)}^{(i)}+\beta_{\left(0, \frac{1}{2}\right)}^{(i) *} \beta_{\left(\frac{1}{3}, \frac{1}{2}\right)}^{(i)}=0 \\
\beta_{\left(\frac{1}{3}, 0\right)}^{(i) *} \beta_{(0,0)}^{(i)}+\beta_{\left(\frac{1}{3}, \frac{1}{2}\right)}^{(i) *} \beta_{\left(\frac{1}{3}, 0\right)}^{(i)}=0 \\
\beta_{\left(\frac{1}{3}, 0\right)}^{(i) *} \beta_{\left(0, \frac{1}{2}\right)}^{(i)}+\beta_{\left(\frac{1}{3}, \frac{1}{2}\right)}^{(i) *} \beta_{\left(\frac{1}{3}, \frac{1}{2}\right)}^{(i)}=D_{i}
\end{array}\right.
$$

and

$$
T=\left(\prod_{i=1}^{\infty} \beta_{\left(c_{i}^{+}, \boldsymbol{l}_{i}\right)}^{(i)}\right)_{H^{+} \times G}
$$

Put

$$
\left\{\begin{array}{l}
T_{r}=\left(\prod_{i=1}^{r} \beta_{\left(c_{i}^{+}, l_{i}\right)}^{(i)}\right)_{H_{r}^{+} \times G_{r}} \\
T_{r}^{*}=\left(\prod_{i=1}^{r} D_{i}\right)^{-1}\left(\prod_{i=1}^{r} \beta_{\left(c_{i}^{*}, l_{i}\right)}^{(i) *}\right)_{H_{r}^{+} \times G_{r}}
\end{array}\right.
$$

for any natural number $r$. Then it follows that

$$
\left\{\begin{array}{l}
T_{r}=\left(\begin{array}{ll}
T_{r-1} \beta_{(0,0)}^{(r)} & T_{r-1} \beta_{\left(0, \frac{1}{2}\right)}^{(r)} \\
T_{r-1} \beta_{\left(\frac{1}{3}, 0\right)}^{(r)} & T_{r-1} \beta_{\left(\frac{1}{3}, \frac{1}{2}\right)}^{(r)}
\end{array}\right) \\
T_{r}^{*}=\left(\begin{array}{ll}
T_{r-1}^{*} \beta_{(0,0)}^{(r) *} & T_{r-1}^{*} \beta_{\left(0, \frac{1}{2}\right)}^{(r) *} \\
T_{r-1}^{*} \beta_{\left(\frac{1}{3}, 0\right)}^{(r) *} & T_{r-1}^{(r) *} \beta_{\left(\frac{1}{3}, \frac{1}{2}\right)}^{(r) *}
\end{array}\right) \cdot D_{r}^{-1}
\end{array}\right.
$$

By (2. 10), (2. 11) and the induction on $r$, it follows that

$$
T_{r}^{*} T_{r}=E_{r}
$$

where $E_{r}$ is the identity matrix of order $2^{r}$. Let $T^{*}$ denote the matrix of which $\left[c^{+}\right] \times[\boldsymbol{l}]$-components $\left(\left[\boldsymbol{c}^{+}\right] \in H^{+},[\boldsymbol{l}] \in G\right)$ are $\left(\prod_{i=1}^{\infty} D_{i}\right)^{-1}\left(\prod_{i=1}^{\infty} \beta_{\left(c_{i}^{*}, \boldsymbol{l}_{i}\right)}^{(i)}\right)$. Here we shall show that the matrix of infinite order $T^{*}$ is well-defined; it is sufficient to show that the infinite product

$$
\prod_{i=1}^{\infty}\left(\vartheta_{[0]}^{(2)}\left(q_{i}\right)-\frac{\vartheta_{\left[\frac{1}{3}\right]}^{(2)}\left(q_{i}\right) \vartheta_{\left[\frac{1}{2}\right]}^{(2)}\left(q_{i}\right)}{\vartheta_{\left[\frac{1}{8}\right]}^{(2)}\left(q_{i}\right)}\right)
$$


is convergent and non-zero. This infinite product (2.13) is absolutely convergent if and only if the infinite sum

$$
\sum_{i=1}^{\infty}\left|\vartheta_{[0]}^{(2)}\left(q_{i}\right)-\frac{\vartheta_{\left[\frac{1}{3}\right]}^{(2)}\left(q_{i}\right) \vartheta_{\left[\frac{1}{2}\right]}^{(2)}\left(q_{i}\right)}{\vartheta_{\left[\frac{1}{6}\right]}^{(2)}\left(q_{i}\right)}-1\right|
$$

is convergent. We may assume that $\left|q_{i}\right|<\frac{1}{2}$ for all $i$. From the inequalities (2.6) and (2.8) we have

$$
\begin{aligned}
& \sum_{i=1}^{\infty}\left|\vartheta_{[0]}^{(2)}\left(q_{i}\right)-\frac{\vartheta_{\left[\begin{array}{l}
1 \\
3
\end{array}\right]\left(q_{i}\right)}^{(2)}\left(\vartheta_{[1]}^{(2)}\right]\left(q_{i}\right)}{\vartheta_{\left[\frac{1}{6}\right]}^{(2)}\left(q_{i}\right)}-1\right| \\
\leq & \sum_{i=1}^{\infty}\left|\vartheta_{[0]}^{(2)}\left(q_{i}\right)-1\right|+\sum_{i=1}^{\infty}\left|\frac{\vartheta_{\left[\frac{1}{3}\right]}^{(2)}\left(q_{i}\right) \vartheta_{\left[\frac{1}{2}\right]}^{(2)}\left(q_{i}\right)}{\vartheta_{\left[\begin{array}{l}
2 \\
6
\end{array}\right]}^{(2)}\left(q_{i}\right)}\right| \\
\leq & \sum_{i=1}^{\infty} \frac{1}{\sqrt{2 s_{i}}}+\sum_{i=1}^{\infty}\left|q_{i}\right|^{\frac{2}{3}} e^{6\left|q_{i}\right|^{\frac{2}{3}}+6+12\left|q_{i}\right|^{\frac{2}{3}}} \\
\leq & \sum_{i=1}^{\infty} \frac{1}{\sqrt{2 s_{i}}}+e^{24} \sum_{i=1}^{\infty} e^{-\frac{2 \pi s_{i}}{3}}
\end{aligned}
$$

where $s_{i}$ are the imaginary parts of $z_{i}$. Therefore the infinite sum (2.14) is convergent, hence the infinite product (2.13) is convergent and non-zero. From (2. 12) it follows that the matrix $T$ is invertible, Q.E.D.

Thus we complete the proof of Proposition 5.

2. 6 Finally we shall show the addition formula in this special case. We start with showing

Lemma 7. The matrix $F$

$$
\left(\prod_{i=1}^{\infty} \vartheta_{\left[c_{i}^{+}\right]+\left[d_{i}^{+}\right]}\left(q_{i}\right) \prod_{i=1}^{\infty} \vartheta_{-\left[c_{i}^{+}\right]+\left[d_{i}^{+}\right]}\left(q_{i}\right)\right)_{H^{+} \times H^{+}}
$$

is invertible.

Proof. For any natural number $i$, put

$$
\begin{aligned}
& \left\{\begin{array}{l}
\gamma_{(0,0)}^{(i)}=\vartheta_{[0]}^{2}\left(q_{i}\right), \quad \gamma_{\left(0, \frac{1}{3}\right)}^{(i)}=\vartheta_{\left[\frac{1}{3}\right]}^{2}\left(q_{i}\right) \\
\gamma_{\left(\frac{1}{3}, 0\right)}^{(i)}=\vartheta_{\left[\frac{1}{3}\right]}^{2}\left(q_{i}\right), \quad \gamma_{\left(\frac{1}{3}, \frac{1}{3}\right)}^{(i)}=\vartheta_{[0]}\left(q_{i}\right) \vartheta_{\left[\frac{1}{3}\right]}\left(q_{i}\right)
\end{array}\right. \\
& \begin{cases}\gamma_{(0,0)}^{(i) *}=\vartheta_{[0]}\left(q_{i}\right) \vartheta_{\left[\frac{1}{3}\right]}\left(q_{i}\right), & \gamma_{\left(0, \frac{1}{3}\right)}^{(i) *}=-\vartheta_{\left[\frac{1}{3}\right]}^{2}\left(q_{i}\right) \\
\gamma_{\left(\frac{1}{3}, 0\right)}^{(i) *}=-\vartheta_{\left[\frac{1}{3}\right]}^{2}\left(q_{i}\right), & \gamma_{\left(\frac{1}{3}, \frac{1}{3}\right)}^{(i) *}=\vartheta_{[0]}^{2}\left(q_{i}\right)\end{cases}
\end{aligned}
$$


and

$$
D_{i}=\operatorname{det}\left(\begin{array}{cc}
\gamma_{(0,0)}^{(i)} & \gamma_{\left(0, \frac{1}{3}\right)}^{(i)} \\
\gamma_{\left(\frac{1}{3}, 0\right)}^{(i)} & \gamma_{\left(\frac{1}{3}, \frac{1}{3}\right)}^{(i)}
\end{array}\right) .
$$

Then we have

$$
F=\left(\prod_{i=1}^{\infty} \gamma_{\left(c_{i}^{+}, d_{i}^{+}\right)}^{(i)}\right)_{H^{+} \times H^{+}}
$$

For any natural number $r$ put

$$
\left\{\begin{array}{l}
F_{r}=\left(\prod_{i=1}^{r} \gamma_{\left(c_{i}^{+}, d_{i}^{+}\right)}^{(i)}\right)_{H_{r}^{+} \times H_{r}^{+}} \\
F_{r}^{*}=\left(\prod_{i=1}^{r} D_{i}\right)^{-1}\left(\prod_{i=1}^{r} \gamma_{\left(c_{i}^{+}, d_{i}^{+}\right)}^{(i) *}\right)_{H_{r}^{+} \times H_{r}^{+}}
\end{array}\right.
$$

Then the similar computation as Lemma. 6 shows that

$$
F_{r}^{*} F_{r}=E_{r} \quad(r=1,2, \cdots)
$$

where $E_{r}$ is the identity matrix of order $2^{r}$. Let $F^{*}$ denote the matrix of which $\left[c^{+}\right] \times\left[d^{+}\right]$-components $\left(\left[c^{+}\right],\left[d^{+}\right] \in H^{+}\right)$are $\left(\prod_{i=1}^{\infty} D_{i}\right)^{-1} \prod_{i=1}^{\infty} \gamma_{\left(c_{i}^{+}, d_{i}^{+}\right) \cdot \text { Here }}^{(i)}$ we shall show that the matrix of infinite order $F^{*}$ is well-defined: it is sufficient to show that the infinite product

$$
\prod_{i=1}^{\infty}\left(\vartheta_{[0]}^{3}\left(q_{i}\right)-\vartheta_{\left[\frac{1}{3}\right]}^{3}\left(q_{i}\right)\right)
$$

is convergent and non-zero. This infinite product is absolutely convergent if and only if the infinite sum

$$
\sum_{i=1}^{\infty}\left|\vartheta_{[0]}^{3}\left(q_{i}\right)-\vartheta_{\left[\frac{1}{3}\right]}^{3}\left(q_{i}\right)-1\right|
$$

is convergent. From (2.5) and (2.6) we have

$$
\begin{aligned}
& \sum_{i=1}^{\infty}\left|\vartheta_{[0]}^{3}\left(q_{i}\right)-\vartheta_{\left[\frac{1}{3}\right]}^{3}\left(q_{i}\right)-1\right| \\
\leq & \sum_{i=1}^{\infty}\left\{\left|\vartheta_{[0]}^{3}\left(q_{i}\right)-1\right|+\left|\vartheta_{\left[\frac{1}{3}\right]}^{3}\left(q_{i}\right)\right|\right\} \\
\leq & \sum_{i=1}^{\infty}\left[\frac{1}{\sqrt{s_{i}}}\left\{\left(1+\frac{1}{\sqrt{s_{i}}}\right)^{2}+\left(1+\frac{1}{\sqrt{s_{i}}}\right)+1\right\}+\left(e^{-\frac{\pi s_{i}}{9}}+\frac{1}{\sqrt{s_{i}}}\right)^{3}\right]
\end{aligned}
$$




$$
\left.\leq \sum_{i=1}^{\infty} 2\left(\frac{1}{\sqrt{s_{i}}}\right)^{3}+6\left(\frac{1}{\sqrt{s_{i}}}\right)^{2}+6\left(\frac{1}{\sqrt{s_{i}}}\right)+e^{-\frac{\pi s_{i}}{3}}\right\}<\infty
$$

Hence the infinite product (2. 16) is convergent and non-zero. From (2. 15) it follows that the matrix $F$ is invertible, Q.E.D.

By the same computation as Proposition 5 we have

Proposttion 7. Let [a] and [b] be in $\boldsymbol{A}$ and $K$ a field such that $\prod_{i=1}^{\infty} \vartheta_{\left[c_{i}^{+}\right]+\left[a_{i}\right]}\left(q_{i} ; u_{i}\right) \prod_{i=1}^{\infty} \vartheta_{-\left[c_{i}^{+}\right]+\left[a^{i}\right]}\left(q_{i} ; u_{i}\right) \quad\left(\left[c^{+}\right] \in H^{+}\right)$and $\prod_{i=1}^{\infty} \vartheta_{\left[c_{i}^{+}\right]+\left[b_{i}\right]}\left(q_{i} ; v_{i}\right) \prod_{i=1}^{\infty} \vartheta_{-\left[c_{i}^{+}\right]+\left[b_{i}\right]}$ $\left(q_{i} ; v_{i}\right)\left(\left[\boldsymbol{c}^{+}\right] \in H^{+}\right)$belong to $\boldsymbol{M}_{K}(2 ; 2[\boldsymbol{a}])$ and $\boldsymbol{M}_{K}(2 ; 2[\boldsymbol{b}])$, respectively. Then $\prod_{i=1}^{\infty} \vartheta_{\left[c_{i}^{+}\right]+\left[a_{i}\right]}\left(q_{i} ; u_{i}\right) \prod_{i=1}^{\infty} \vartheta_{-\left[c_{i}^{+}\right]+\left[a_{i}\right]}\left(q_{i} ; u_{i}\right) \prod_{i=1}^{\infty} \vartheta_{\left[d_{i}^{+}\right]+\left[b_{i}\right]}\left(q_{i} ; v_{i}\right) \prod_{i=1}^{\infty} \vartheta_{-\left[d_{i}^{+}\right]+\left[b_{i}\right]}\left(q_{i} ; v_{i}\right)$ $\left(\left[c^{+}\right],\left[\boldsymbol{d}^{+}\right] \in H^{+}\right)$

form a base of $\boldsymbol{M}_{K}(2 \times 2 ; 2[\boldsymbol{a}] \times 2[\boldsymbol{b}])$.

TheOREM (The ADDITION FORMULA)

$$
\begin{aligned}
& \prod_{i=1}^{\infty} \vartheta_{\left[a_{i}\right]+\left[b_{i}\right]}\left(q_{i} ; u_{i} v_{i}\right) \prod_{i=1}^{\infty} \vartheta_{\left[a_{i}\right]-\left[b_{i}\right]}\left(q_{i} ; u_{i} v_{i}^{-1}\right) \\
= & \sum_{\left[\boldsymbol{c}^{+}\right],\left[\boldsymbol{d}^{+}\right] \in H^{+}} \boldsymbol{\alpha}_{\left(\boldsymbol{c}^{+}, \boldsymbol{d}^{+}\right)} \prod_{i=1}^{\infty} \vartheta_{\left[c_{i}^{+}\right]+\left[a_{i}\right]}\left(q_{i} ; u_{i}\right) \prod_{i=1}^{\infty} \vartheta_{-\left[c_{i}^{+}\right]+\left[a_{i}\right]}\left\langle q_{i} ; u_{i}\right) \prod_{i=1}^{\infty} \vartheta_{\left[d_{i}^{+}\right]+\left[b_{i}\right]}\left(q_{i} ; v_{i}\right) \\
& \prod_{i=1}^{\infty} \vartheta_{-\left[d_{i}^{+}\right]+\left[b_{i}\right]}\left(q_{i} ; v_{i}\right) \quad([\boldsymbol{a}],[\boldsymbol{b}] \in \boldsymbol{A})
\end{aligned}
$$

where $\left(\boldsymbol{\alpha}_{\left(\boldsymbol{c}^{+}, \boldsymbol{d}^{+}\right)}\right)$is the inverse matrix of the matrix $\left.{ }^{5}\right)$

$$
\left(\prod_{i=1}^{\infty} \vartheta_{\left[c_{i}^{+}\right]+\left[d_{i}^{+}\right]}\left(q_{i}\right) \prod_{i=1}^{\infty} \vartheta_{-\left[c_{i}^{+}\right]+\left[d_{i}^{+}\right]}\left(q_{i}\right)\right)_{H^{+} \times H^{+}}
$$

Proof. By virtue of Lemma 1 and Proposition 7 we have

$$
\begin{gathered}
\prod_{i=1}^{\infty} \vartheta_{\left[a_{i}\right]+\left[b_{i}\right]}\left(q_{i} ; u_{i} v_{i}\right) \prod_{i=1}^{\infty} \vartheta_{\left[a_{i}\right]-\left[b_{i}\right]}\left(q_{i} ; u_{i} v_{i}^{-1}\right) \\
=\sum_{\left[\boldsymbol{c}^{+}\right],\left[\boldsymbol{d}^{+}\right] \in H^{+}} \lambda_{\left(\boldsymbol{c}^{+}, \boldsymbol{d}^{+}\right)} \prod_{i=1}^{\infty} \vartheta_{\left[c_{i}^{+}\right]+\left[a_{i}\right]}\left(q_{i} ; u_{i}\right) \prod_{i=1}^{\infty} \vartheta_{-\left[c_{i}^{+}\right]+\left[a_{i}\right]}\left(q_{i} ; u_{i}\right) \prod_{i=1}^{\infty} \vartheta_{\left[d_{i}^{+}\right]+\left[b_{i}\right]}\left(q ; v_{i}\right) \\
\prod_{i=1}^{\infty} \vartheta_{-\left[d_{i}^{+}\right]+\left[b_{i}\right]}\left(q_{i} ; v_{i}\right) .
\end{gathered}
$$

Putting $u_{i}=q_{i}$ and $v_{i}=q_{i}(i=1,2, \cdots)$, we have

5) The existence of the inverse matrix of this matrix is guaranteed by Lemma 7 . 


$$
\lambda_{\left(\boldsymbol{c}^{+}, \boldsymbol{d}^{+}\right)}=\left(\prod_{i=1}^{\infty} \vartheta_{\left[c_{i}^{+}\right\rceil+\left[d_{i}^{+}\right]}\left(q_{i}\right) \prod_{i=1}^{\infty} \vartheta_{-\left[c_{i}^{+}\right]+\left[d_{i}^{+}\right]}\left(q_{i}\right)\right)^{-1} .
$$

Here we shall show that the right hand double series of the addition formula is absolutely convergent in any bounded domain of values of each variable $z_{i}$ and $w_{i}$, where $u_{i}=e^{\pi \sqrt{-1} z_{i}}$ and $v_{i}=e^{\pi \sqrt{-1}} w_{i}(i=1,2, \cdots \cdots)$. Put

$$
\prod_{i=1}^{\infty} \vartheta_{\left[a_{i}\right]+\left[b_{i}\right]}\left(q_{i} ; u_{i} v_{i}\right) \prod_{i=1}^{\infty} \vartheta_{\left[a_{i}\right]-\left[b_{i}\right]}\left(q_{i} ; u_{i} v_{i}^{-1}\right)=\sum_{\left[\mathbf{c}^{+}\right],\left[\boldsymbol{d}^{+}\right] \in H^{+}} \varphi_{\left(\mathbf{c}^{+}, \boldsymbol{d}^{+}\right)}
$$

Let all variables $z_{i}$ and $w_{i}(i=1,2, \cdots)$ lie in one and the same bounded domain. Then by virtue of the proof of Lemma 7 and (2.7) we have

$$
\left|\varphi_{\left(\boldsymbol{c}^{+}, \boldsymbol{d}^{+}\right)}\right| \leq C \frac{\prod_{i=1}^{N}\left|\gamma_{\left(c_{i}^{(i)}, d_{i}^{+}\right)}^{(i)}\right| \prod_{i=1}^{\infty}\left|\vartheta_{[0]}\left(q_{i}\right)\right|}{\prod_{i=1}^{N}\left|\vartheta_{\left[\frac{1}{3}\right]}\left(q_{i}\right)\right|} \prod_{i=1}^{\infty}\left|q_{i}\right|^{2\left\{\left(c_{i}^{+}\right)^{2}+\left(d_{i}^{+}\right)^{2}\right\}}
$$

where $N$ is an integer such that $c_{i}^{+}=d_{i}^{+}=0$ for all $i>N$, and $C$ is a constant depending only on $[\boldsymbol{a}]$ and $[\boldsymbol{b}]$. Put

$$
\varphi_{\left(\boldsymbol{c}^{+}, \boldsymbol{d}^{+}\right)}(i)= \begin{cases}\gamma_{\left(c_{i}^{+}, d_{i}^{+}\right)}^{(i) *}\left(\vartheta_{\left[\frac{1}{3}\right]}\left(q_{i}\right)\right)^{-1}\left|q_{i}\right|^{2\left\{\left(c_{i}^{+}\right)^{2}+\left(d_{i}^{+}\right)^{2}\right\}} & (i \leq N) \\ \vartheta_{[0]}\left(q_{i}\right)\left|q_{i}\right|^{\left.2\left\{c_{i}^{+}\right)^{2}+\left(d_{i}^{+}\right)^{2}\right\}} & (i>N+1) .\end{cases}
$$

Here we may assume that $\left|q_{i}\right|^{\frac{1}{3}}<\frac{1}{2}$ for all $i$. Then from the inequalities (2. 8) it follows that for all $i$

$$
\begin{cases}\left|\varphi_{\left(\boldsymbol{c}^{+}, \boldsymbol{d}^{+}\right)}(i)\right| \leq e^{24\left|q_{i}\right|^{\frac{1}{3}}} & \left(c_{i}^{+}+d_{i}^{+}=0\right) \\ \left|\varphi_{\left(\boldsymbol{c}^{+}, \boldsymbol{d}^{+}\right)}(i)\right| \leq\left|q_{i}\right|^{\frac{1}{3}} e^{24\left|\boldsymbol{q}_{i}\right|^{\frac{1}{3}}} & \left(c_{i}^{+}+d_{i}^{+} \neq 0\right) .\end{cases}
$$

Expanding the integers in the 2-adic numbers, we can identify $H^{+}$with the set $\{0,1,2, \cdots \cdots\}$ consisting non-negative integers. Put

$$
\sum_{\left[c^{+}\right],\left[d^{+}\right] \in H^{+}}\left|\varphi_{\left(c^{+}, d^{+}\right)}\right|=\sum_{k, l=0}^{\infty}\left|\varphi_{(k, l)}^{*}\right| .
$$

Consider the infinite series $\sum_{m=0}^{\infty} \sigma_{m}$ defined by

$$
\begin{aligned}
& \sigma_{0}=\left|\varphi_{(0,0)}^{*}\right| \\
& \sigma_{1}=\left|\varphi_{(1,0)}^{*}\right|+\left|\varphi_{(1,1)}^{*}\right|+\left|\varphi_{(0,1)}^{*}\right| \\
& \vdots \\
& \sigma_{m}=\left|\varphi_{(m, 0)}^{*}\right|+\left|\varphi_{(m, 1)}^{*}\right|+\left|\varphi_{(m, 2)}^{*}\right|+\cdots+\left|\varphi_{(1, m)}^{*}\right|+\left|\varphi_{(0, m)}^{*}\right| \\
& \vdots
\end{aligned}
$$


It is well-known that the series $\sum_{m=0}^{\infty} \sigma_{m}$ is convergent if and only if the double series $\sum_{\left[c^{+}\right],\left[d^{+}\right] \in H^{+}}\left|\varphi_{\left(c^{+}, d^{+}\right)}\right|=\sum_{k, l=0}^{\infty}\left|\varphi_{(k, l)}^{*}\right|$ is convergent. By virtue of the inequalities (2.17) we have

$$
\begin{aligned}
& \sigma_{0}=e^{24} \sum_{i=1}^{\infty}\left|q_{i}\right|^{\frac{1}{3}} \\
& \sigma_{m}=(1+2 m)\left|q_{i}\right|^{\frac{1}{3}} e^{24} \sum_{i=1}^{\infty}\left|q_{i}\right|^{\frac{1}{3}} \quad\left(2^{j-1} \leq m<2^{j} ; j=1,2, \cdots \cdots\right) .
\end{aligned}
$$

Therefore

$$
\sum_{m=0}^{\infty} \sigma_{m}=e^{24} \sum_{i=1}^{\infty}\left|q_{i}\right|^{\frac{1}{3}}\left(1+\sum_{j=1}^{\infty} 2^{j} 2^{j-i} e^{-\frac{\pi s_{j}}{3}}\right)
$$

where $s_{j}$ are the imaginary parts of $\tau_{j}$. Since the series $\sum_{j=1}^{\infty} \frac{1}{\sqrt{s_{j}}}$ is convergent, the series

$$
\sum_{i=1}^{\infty}\left|q_{i}\right|^{\frac{1}{3}}=\sum_{i=1}^{\infty} e^{-\frac{\pi s_{i}}{3}}
$$

and

$$
\sum_{j=1}^{\infty} 2^{2 j-1} e^{-\frac{\pi s_{j}}{3}}
$$

are convergent. Hence the double series $\sum_{\left[\boldsymbol{c}^{+}\right],\left[\boldsymbol{d}^{+}\right] \in H^{+}}\left|\varphi_{\left(\boldsymbol{c}^{+}, \boldsymbol{d}^{+}\right)}\right|$is convergent, which completes the proof of Theorem, Q.E.D.

2. 7 Let $\tau_{i, j}(i, j=1,2, \cdots \cdots)$ be complex numbers such $\tau_{i, j}=\tau_{j, i}$, and $z_{i}(i=1,2, \cdots \cdots)$ be complex variables. For the sake of simplicity we shall use the matrices notations $\tau=\left(\tau_{i, j}\right)$ and $z=\left(z_{1}, z_{2}, \cdots \cdots\right)$. In [6] we introduced the theta functions of variables $z$ with rational characteristics $[\boldsymbol{a}]$ :

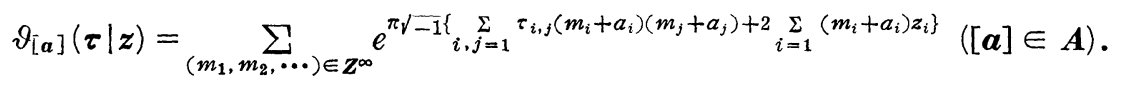

We showed the sufficient conditions under which these theta functions are absolutely convergent. ${ }^{6}$ ) Then for these theta functions is an addition formula realized?

6) See Proposition 2 and 3 in [6]. 


\section{REFERENCES}

[1] R. Bellman, A brief introduction to theta functions, New York, 1961.

[2] A. Krazer, Lehrbuch der Thetafunktionen, Leipzig, 1903.

[3] H. Morikawa, On the explicite defining relations of abelian schemes of level three, Nagoya Math. J., Vol. 27 (1966), p. 143-157.

[4] H. Morikawa, On the defining equations of abelian varieties, Nagoya Math. J., Vol. 30 (1967), p. 143-162.

[5] D. Mumford, On the equations defining abelian varieties, I, II, III, Inv. Math., Vol. 1 (1966) p. 287-354, Vol. 3 (1967) p. 75-135, p. 215-244.

[6] K. Tahara, On the hyperelliptic Riemann surfaces of infinite genus with absolutely convergent Riemann's theta functions, (to appear in Nagoya Math. J.)

[ 7 ] E.T. Whittaker and G.N. Watson, A course of modern analysis, (fourth edition) Cambridge, 1958.

Nagoya University 